\title{
Community norms for the eating disorder examination questionnaire (EDE-Q) among cisgender bisexual plus women and men
}

\author{
Jason M. Nagata ${ }^{1}$ (D) Emilio J. Compte ${ }^{2,3} \cdot$ Stuart B. Murray $^{4} \cdot$ Rebecca Schauer $^{1} \cdot$ Erica Pak $^{1} \cdot$ Annesa Flentje $^{5,6,8}$. \\ Matthew R. Capriotti ${ }^{7,8} \cdot$ Micah E. Lubensky ${ }^{5,8} \cdot$ Mitchell R. Lunn ${ }^{8,9,10} \cdot$ Juno Obedin-Maliver ${ }^{8,10,11}$
}

Received: 8 July 2020 / Accepted: 10 November 2020 / Published online: 3 December 2020

(C) The Author(s) 2020

\begin{abstract}
Purpose Cisgender bisexual plus (including bisexual, pansexual, and polysexual) women and men experience unique health concerns including eating disorders. The purpose of this study was to develop community norms for eating disorder attitudes and disordered eating behaviors in cisgender bisexual plus women and men using the Eating Disorders Examination Questionnaire (EDE-Q).

Methods Participants were cisgender bisexual plus women $(n=462)$ and men $(n=93)$ participants in The PRIDE Study, an existing study of sexual and gender minority people.

Results Mean and standard deviation of EDE-Q scores among cisgender bisexual plus women and men, respectively, were: Global (1.75 $\pm 1.26,1.56 \pm 1.18)$, Restraint $(1.34 \pm 1.44,1.42 \pm 1.53)$, Eating Concern $(0.96 \pm 1.13,0.63 \pm 0.96)$, Weight Concern $2.27 \pm 1.55,1.89 \pm 1.46$ ), and Shape Concern $42 \pm 1.62,2.30 \pm 1.57)$. Among cisgender bisexual plus women and men, respectively, $27.5 \%$ and $22.6 \%$ scored in the clinically significant range on the Global score. Bisexual plus women and men reported any occurrence $(\geq 1 / 28$ days) of dietary restraint $(19.3 \%, 23.7 \%)$, objective binge episodes $(11.1 \%, 10.8 \%)$, excessive exercise $(4.5 \%, 5.4 \%)$, self-induced vomiting $(1.7 \%, 0.0 \%)$, and laxative misuse $(0.4 \%, 1.1 \%)$, respectively. A lower percentage of age-matched cisgender bisexual plus women (18-25 years) reported any occurrence of objective binge episodes, self-induced vomiting, laxative misuse, and excessive exercise than previously published in young women. Agematched cisgender bisexual plus men (18-26 years) reported higher weight concern subscale scores than previously published in young men.
\end{abstract}

Conclusions These norms should aid clinicians in applying and interpreting the EDE-Q scores of cisgender bisexual plus women and men.

Level of evidence Level V: cross-sectional descriptive study

Keywords Eating disorder $\cdot$ Bisexual $\cdot$ Cisgender $\cdot$ Norms $\cdot$ Sexual minorities

The article is part of the Topical Collection on Males and eating and weight disorders.

Jason M. Nagata

jasonmnagata@gmail.com

1 Department of Pediatrics, University of California, San Francisco, 550 16th Street, 4th Floor, Box 0110, San Francisco, CA 94158, USA

2 School of Psychology, Universidad Adolfo Ibáñez, Santiago, Chile

3 Comenzar de Nuevo Treatment Center, Monterrey, México

4 Department of Psychiatry and Behavioral Sciences, University of Southern California, Los Angeles, CA, USA

5 Department of Community Health Systems, University of California, San Francisco, San Francisco, CA, USA
6 Alliance Health Project, Department of Psychiatry and Behavioral Sciences, University of California, San Francisco, CA, USA

7 Department of Psychology, San José State University, San Jose, CA, USA

8 The PRIDE Study/PRIDEnet, Stanford University School of Medicine, Stanford, CA, USA

9 Division of Nephrology, Department of Medicine, Stanford University School of Medicine, Stanford, CA, USA

10 Department of Epidemiology and Population Health, Stanford University School of Medicine, Stanford, CA, USA

11 Department of Obstetrics and Gynecology, Stanford University School of Medicine, Stanford, CA, USA 


\section{Introduction}

Cisgender (i.e., those whose gender identity matches what is commonly associated with the sex assigned to them at birth) bisexual women and men experience unique health disparities in physical, mental, and sexual health outcomes [1]. In addition to bisexuality, other plurisexual identities (i.e., romantic or sexual attraction and/ or behavior with members of more than one sex or gender)—such as pansexuality or polysexuality - are traditionally underrepresented in research [2]. In this paper, we refer to people who identify as bisexual, pansexual, or polysexual as "bisexual plus." Bisexual plus people may experience prejudice, stigma, and discrimination based on sexual orientation from heterosexual communities, in response to which they may experience sexual minority stress and disordered eating [3-5]. While a growing literature identified disordered eating behaviors in sexual minority (i.e., non-heterosexual) populations, most investigations have grouped gay, lesbian, and bisexual plus populations together [6] and few focus exclusively on cisgender bisexual plus people.

There is little research on eating disorder norms for bisexual plus women [7]. One study found that bisexual women typically engage in more body checking (e.g., an individual's tendency to repeatedly check their weight and shape through ritualistic weighing, compulsive mirror checking, and using the fit of clothes to judge weight changes) than heterosexual women [8]. Despite this group difference in body image observations, Henn et al. [8] reported no accordant evidence of a different likelihood or vulnerability to body image disturbances and eating disordered behaviors based on sexual orientation [8]. However, in another study, $10.6 \%$ of bisexual women reported both subjective binge eating and compensatory behavior compared to only $2.8 \%$ of lesbian women [9].

Research on eating disorder attitudes and behaviors in cisgender bisexual plus men is sparse [10, 11]. The existing research in presumably cisgender men suggests that bisexual men experience greater dissatisfaction with their bodies and a higher incidence of eating disorder symptoms compared to heterosexual men [12]. One study comparing rates of disordered eating behaviors among university men found the highest rates of objective binge eating among men who have sex with either men or women (29\%) compared to men who have sex with men $(6 \%)$ or men who have sex with women (10\%) [13]. Among bisexual men, exercise frequency has been found to be significantly associated with eating disorder symptomology, suggesting that, when compared with any other subgroup, bisexual men are more likely to use exercise as a method of weight control [14].

Although eating disorder attitudes and behaviors have been noted to be higher in presumably cisgender and heterosexual women than men $[15,16]$, gender differences are unknown among cisgender bisexual plus women and men. Given higher eating disorder symptoms in bisexual compared to heterosexual men [12], gender differences among bisexual plus women and men may be attenuated.

This study aims to (1) develop community norms for eating disorder attitudes and disordered eating behaviors using the EDE-Q among cisgender bisexual plus women and men, (2) compare community norms of the EDE-Q among cisgender bisexual plus women (age-matched) with those previously published in presumed cisgender heterosexual young women [16], and (3) compare community norms of the EDE-Q among cisgender bisexual plus men (age-matched) with those previously published in presumed cisgender heterosexual young men [15]. We hypothesized that EDE-Q norms would be higher in cisgender bisexual plus women and men than those previously published in presumed cisgender heterosexual young women [16] and men [15], respectively.

\section{Methods}

\section{Study design and population}

This study uses cross-sectional data from The Population Research in Identity and Disparities for Equality (PRIDE) Study, a national longitudinal cohort study of sexual and gender minority (SGM) adults in the US which include, but are not limited to, people who identify as lesbian, gay, bisexual, transgender, and/or queer. This ancillary study invited all The PRIDE Study participants to participate in a crosssectional, self-reported, online survey from April to August 2018. The PRIDE Study engages, recruits, and consents participants. It also collects data on a web-based platform that is accessible from any mobile device or computer with Internet access (www.pridestudy.org). Associated with The PRIDE Study is PRIDEnet, a national network of organizations and individuals created to actively engage SGM communities in research for and beyond The PRIDE Study. Participants in The PRIDE Study are recruited through PRIDEnet constituents; social media advertising; digital communications (blog posts, newsletters); distribution of The PRIDE Studybranded promotional items; direct outreach at conferences, symposiums, and events; and word-of-mouth. Inclusion criteria included age $\geq 18$ years, self-identification as a SGM person, residing in the US or its territories, and written and oral proficiency in English to read and answer questions. Further description of the cohort population, demographics, and technology that supports the study have been previously described $[17,18]$. This study was approved by the University of California, San Francisco Institutional Review Board on 2 February 2018 (\#16-21213), as well as The 
PRIDE Study's Research Advisory and Participant Advisory Committees.

We included cisgender bisexual plus women and men in this analysis. Participants were asked about their gender identity ("What is your current gender identity?") and the sex assigned to them at birth ("What sex were you assigned at birth on your original birth certificate?"). Cisgender women were defined as persons who responded "woman" for gender identity and "female" for sex assigned at birth. Cisgender men were defined as persons who responded "man" for gender identity and "male" for sex assigned at birth. Participants who indicated sexual orientation as bisexual ( $n=388$ women, 82 men), pansexual $(n=221$ women, 32 men), or polysexual ( $n=3$ women, 0 men, write-in) were included as bisexual plus. Participants could indicate more than one sexual orientation. Participants who reported multiple gender identities or sexual orientations other than bisexual, pansexual, or polysexual (such as gay, lesbian, or queer) were excluded. Of the 4285 participants from The PRIDE Study who completed the questionnaire, 507 were cisgender bisexual plus women and 100 were cisgender bisexual plus men. Participants who fully completed all of the EDE-Q questions (462 cisgender bisexual plus women and 93 cisgender bisexual plus men) were included in the current study. In addition, data from 978 cisgender gay men ( $M$ age $=42.0, \mathrm{SD}=15.1$, range 18 to 82 years) and 476 cisgender lesbian women from previously published reports of The PRIDE Study $[19,20]$ were considered for group comparisons across genders. For the purpose of this study, only participants with no missing values in the EDE-Q were considered.

\section{Measures}

The EDE-Q is a self-report questionnaire that assesses disordered eating attitudes and behaviors over the previous 28 days [21]. The measure provides four attitudinal subscale scores: Restraint (5 items), Eating Concern (5 items), Shape Concern (8 items), and Weight Concern (5 items). An overall Global score is the mean of the four subscale scores. Responses are on a 7-point ordinal response; higher scores reflect greater eating-related concerns or behaviors. Frequencies of disordered eating behaviors (e.g., binge eating, compensatory behaviors) are assessed. Cronbach's alpha in this study for cisgender bisexual plus women and men, respectively, were as follows: Global score (0.94 and 0.93 ), Restraint (0.79 and 0.84), Eating Concern (0.82 and $0.81)$, Weight Concern (0.84 and 0.83), and Shape Concern $(0.91$ and 0.89$)$. A cutoff of $>2.48$ as a marker of clinical significance (range 0-6; higher scores indicate greater symptoms) on the Global score was utilized based on a cut point with maximum sensitivity and specificity as determined by receiver operator characteristics (ROC) curve analysis for the 28-item EDE-Q established by Machado et al. (2020) [22].

In the EDE-Q, the frequency of binge eating and compensatory behaviors were assessed by the number of episodes occurring during the past 28 days, in accordance with previous literature $[15,16,19,20,23-27]$ to allow comparisons across studies. Any occurrence was defined as $\geq 1$ episode in the past 28 days $[15,16]$. Regular occurrences of dietary restraint were defined as going for long periods of time ( $\geq 8 \mathrm{~h}$ ) without eating anything to influence shape or weight for $\geq 13$ days over the past 28 days as previously defined [15, 16, 28]. The choice of the cut-off for dietary restraint corresponded to an average of $>3$ days per week. Thus, the cutoff was based on $\geq 3$ on the $0-6$ scale of the EDE-Q item 2. A rating of 4 on items/subscales of the EDE-Q has been used as a cut-off for clinical severity $[15,16]$, so the selection of the cut-off corresponding to a rating of $\geq 3$ allowed for inclusion of a somewhat lower frequency given that the fasting behavior represented an extreme form of dietary restraint $[15,28]$. The cutoff is also consistent with the clinical cutoff of $>2$ identified by Machado et al. [22]. Regular occurrences of excessive exercise were defined as exercising in a driven or compulsive way as a means of controlling weight, shape or amount of fat, or burning off calories for $\geq 20$ days over the past 28 days $[15,16]$. The choice of the cutoff for driven or compulsive exercise was based on an average of $\geq 5$ days per week, and this higher threshold was selected to enhance the likelihood that the cut-off reflects clinical severity, given that the item may not clearly distinguish between pathological and adaptive forms of exercise $[15,16]$. For all other behaviors (objective binge episodes, self-induced vomiting, and laxative misuse), regular occurrence was defined as $\geq 4$ occurrences over the past 28 days [15, 16], which would average to at least one episode per week and is consistent with the current Diagnostic and Statistical Manual, 5th Edition (DSM-5) criteria for bulimia nervosa and binge eating disorder [15, 16, 29].

Demographic information (age, race, ethnicity, and education), weight, and height were based on self-report. The standard formula weight (kilograms) divided by height (meters) squared was used to determine body mass index $\left(\mathrm{BMI}=\right.$ weight $/$ height $\left.^{2}\right)$. Participants were also asked: "Has a mental health professional or physician ever told you that you have an eating disorder such as anorexia nervosa, bulimia nervosa, or binge eating disorder?" If affirmative, participants were asked to specify which type (participants could select more than one option). Options included anorexia nervosa, bulimia nervosa, binge eating disorder, or other/not specified. 


\section{Data analysis}

SPSS 20.0 was used for all analyses. Data are presented as mean (SD), median (IQR), and percentage. Associations between participant's BMI and EDE-Q subscales and global scores were assessed through Pearson productmoment correlations coefficient; values of $r>0.10$ were considered weak, $r>0.30$ were considered moderate, and $r>0.50$ were considered strong correlations [30]. The Student $-t$ test was used for group comparisons across EDE-Q subscales and Global score, and Z-tests (or Fisher's exact tests) were conducted for comparisons across the proportions of individuals who reported each ED behavior. We assessed group comparisons of norms in the present sample of cisgender bisexual plus women to norms for cisgender lesbian women previously published from The PRIDE Study [20]. We also assessed group comparisons of norms in the present sample of cisgender bisexual plus men to norms for cisgender gay men previously published from The PRIDE Study [19]. In addition, we selected previously published norms of presumed cisgender heterosexual women [16] and men [15] as comparison groups as they were samples that most closely matched The PRIDE Study's samples (e.g., USbased, non-clinical, community samples of adults). These samples were presumed to be predominantly cisgender and heterosexual as comprehensive gender identity and sexual orientation were not assessed $[15,16]$. These previously published samples were of young adults; thus, we analyzed a subset of age-matched cisgender bisexual plus individuals for comparison. We calculated norms in a subset $(N=144)$ of cisgender bisexual plus women from The PRIDE Study (ages 18-25 years) to compare norms with those previously published in presumed cisgender heterosexual young adult women [16]. We calculated norms in a subset $(N=17)$ of cisgender bisexual plus men from The PRIDE Study (ages 18-26 years) to compare norms with those previously published in presumed cisgender heterosexual young adult men [15]. Finally, given the significant gender differences that were previously reported in norms of presumed cisgender heterosexual undergraduate women and men $[15,16]$, we assessed for gender differences in the norms among agematched participants in our samples of cisgender bisexual plus men and women. Two-tailed tests with an adjusted $p^{-}$ value (Bonferroni) were set at 0.005 for significance.

\section{Power calculations}

Given a sample size of presumed cisgender heterosexual women $(n=723)$ and cisgender bisexual plus women $(n=144)$ [16], and using an estimated mean for EDE-Q Global Score $(1.74 \pm 1.30)$, our study had statistical power (alpha $=0.05$, two-sided) of $>80 \%$ to detect a 0.33 difference in EDE-Q Global Score. Given a sample size of presumed cisgender heterosexual men $(n=404)$ and cisgender bisexual plus men $(n=17)$ [15], and using an estimated mean for EDE-Q Global Score $(1.09 \pm 1.00)$, our study had statistical power (alpha $=0.05$, two-sided) of $>80 \%$ to detect a 0.61 difference in EDE-Q Global Score.

\section{Results}

\section{Cisgender bisexual plus women}

A total of 462 cisgender bisexual plus women participated in this study (Table 1). The mean age was 31.9 years $(\mathrm{SD}=9.4)$ ranging from 18 to 71 years of age. The mean BMI was $28.8 \mathrm{~kg} / \mathrm{m}^{2}(\mathrm{SD}=8.2)$. A total of $82.0 \%$ of the participants identified as White (non-Hispanic/Latino), $4.8 \%$ as Hispanic/Latino, 3.0\% as Asian or Pacific Islander (nonHispanic/Latino), $2.6 \%$ as Black (non-Hispanic/Latino), $0.2 \%$ as Native American, and $7.6 \%$ as other or multiracial. In addition, $79.6 \%$ of participants had completed a college degree or higher. Overall, $11.3 \%$ of participants reported having ever been told by a mental health provider or physician that they had an eating disorder, including anorexia nervosa (5.4\%), bulimia nervosa (3.2\%), binge eating disorder $(3.7 \%)$, or another or unspecified eating disorder (3.4\%).

Mean scores, standard deviations, and percentile ranks for the EDE-Q subscales and Global Score are presented in Table 2. Among cisgender bisexual plus women, 5.9\% scored in the clinically significant range on the Restraint, $3.9 \%$ on the Eating Concern, $19.0 \%$ on the Weight Concern, $19.7 \%$ on the Shape Concern subscales, and $5.8 \%$ on the Global score scale. In addition, $27.5 \%$ of cisgender bisexual

Table 1 Demographic characteristics of cisgender bisexual plus women $(N=462)$ and men $(N=93)$ from The PRIDE Study

\begin{tabular}{|c|c|c|}
\hline & $\begin{array}{l}\text { Cisgender } \\
\text { bisexual plus } \\
\text { women }\end{array}$ & $\begin{array}{l}\text { Cisgender } \\
\text { bisexual plus } \\
\text { men }\end{array}$ \\
\hline$N$ & 462 & 93 \\
\hline Demographic characteristics & Mean $\pm \mathrm{SD} / \%$ & Mean $\pm \mathrm{SD} / \%$ \\
\hline Age, years & $31.9 \pm 9.4$ & $38.3 \pm 12.8$ \\
\hline \multicolumn{3}{|l|}{ Race/ethnicity } \\
\hline White & $82.0 \%$ & $79.6 \%$ \\
\hline Hispanic/Latino & $4.8 \%$ & $2.2 \%$ \\
\hline Asian/Pacific Islander & $3.0 \%$ & $7.5 \%$ \\
\hline Black/African American & $2.6 \%$ & $2.2 \%$ \\
\hline Native American & $0.2 \%$ & $0.0 \%$ \\
\hline Multiracial/Other & $7.6 \%$ & $8.5 \%$ \\
\hline \multicolumn{3}{|l|}{ Educational attainment } \\
\hline College degree or higher & $79.6 \%$ & $80.2 \%$ \\
\hline Body mass index (BMI), $\mathrm{kg} / \mathrm{m}^{2}$ & $28.8 \pm 8.2$ & $28.1 \pm 7.2$ \\
\hline
\end{tabular}




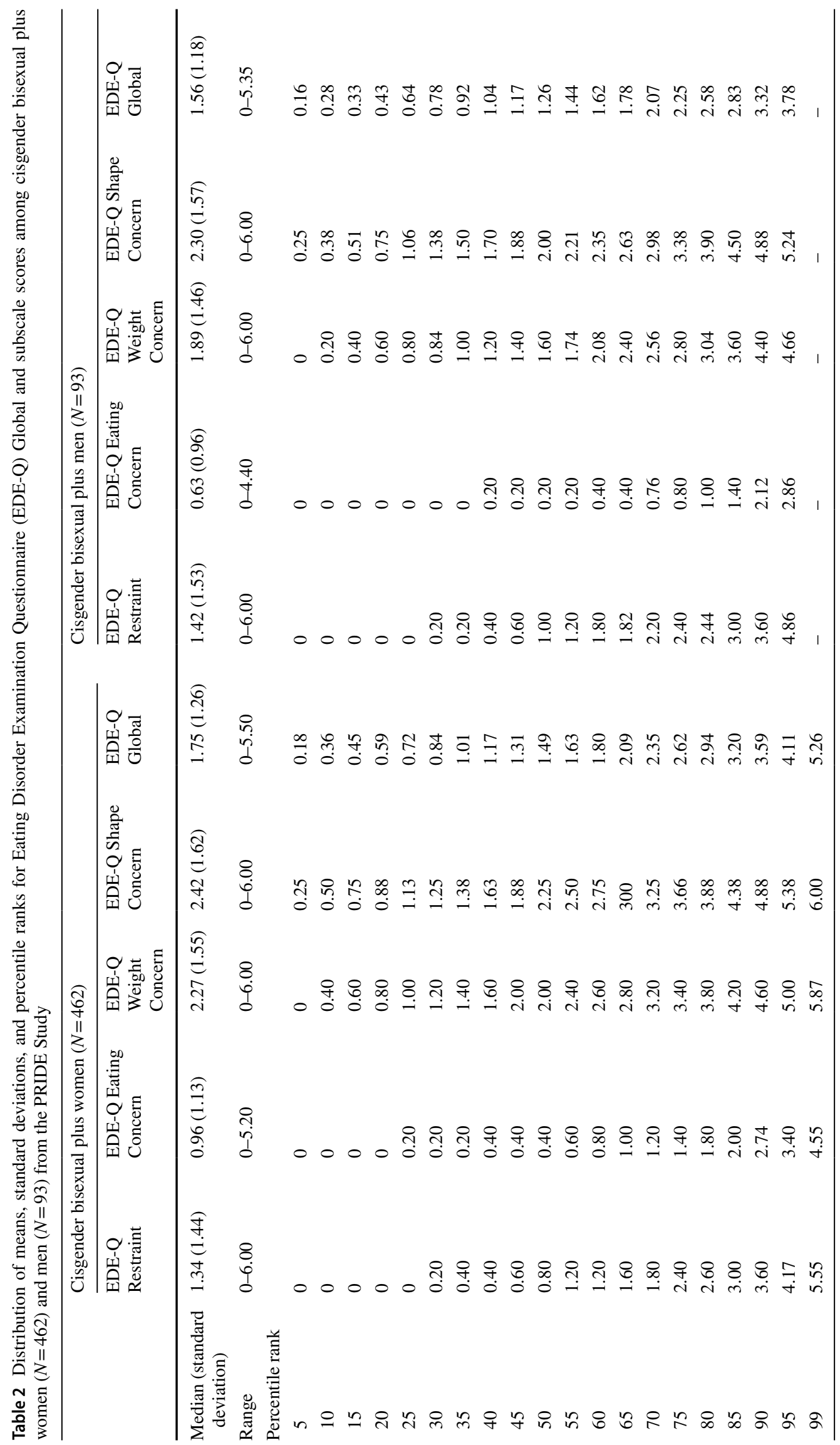


plus women scored above the 2.48 cutoff score suggested by Machado et al. (2020) [22].

BMI was significantly positively correlated with all the subscales and the Global score of the EDE-Q. Significant weak correlations were observed between participants' BMI and the Eating Concern $(r=0.22, p<0.001)$, and Shape Concern $(r=0.29, p<0.001)$ subscales, and the Global score $(r=0.28, p<0.001)$. A moderate correlation was observed between BMI and the Weight Concern $(r=0.36, p<0.001)$. No significant correlation was observed between participants' BMI and the Restraint subscale ( $r=0.07, p=0.141)$.

Any occurrence and regular occurrences of key disordered eating behaviors are presented in Table 3. At least one episode of dietary restraint during the previous 28 days was observed for almost $20 \%$ of cisgender bisexual plus women. Of the total sample, more than $10 \%$ of the participants endorsed at least one episode of objective binge eating, and almost 5\% reported at least one episode of excessive exercise over the past 28 days. Episodes of self-induced vomiting $(1.7 \%)$ and laxative misuse $(0.4 \%)$ in the last 28 days were rarely observed.

Table 4 shows differences in the EDE-Q subscales, the EDE-Q Global score, and disordered eating behaviors between cisgender bisexual plus women and cisgender lesbian women. No significant differences were observed in either attitudinal subscales or disordered eating behaviors in these two groups.

Table 5 shows group comparison in EDE-Q subscales, Global score, and disordered eating behaviors between agematched cisgender bisexual plus women from The PRIDE Study with presumed cisgender heterosexual women from previously published norms [16]. No significant differences were observed between cisgender bisexual plus women and cisgender women in the attitudinal subscales or the Global score of the EDE-Q. However, a higher percentage of presumed cisgender heterosexual women reported any occurrence of objective binge episodes, self-induced vomiting, laxative misuse, and excessive exercise than cisgender bisexual plus women. No significant differences were observed in occurrence of dietary restraint between cisgender bisexual plus women and cisgender women [16].

\section{Cisgender bisexual plus men}

A total of 93 cisgender bisexual plus men participated in this study (Table 1). The mean age was 38.3 years old $(\mathrm{SD}=12.8)$ in a range of 20 to 76 years of age. The mean BMI was $28.1 \mathrm{~kg} / \mathrm{m}^{2}(\mathrm{SD}=7.2)$. A total of $79.6 \%$ of the participants identified as White (non-Hispanic/Latino), $7.5 \%$ as Asian or Pacific Islander (non-Hispanic/Latino), $2.2 \%$ as Black (non-Hispanic/Latino), 2.2\% as Hispanic/ Latino, and $8.5 \%$ as other or Multiracial. In addition, $80.2 \%$ of participants had completed a college degree or higher. Overall, $3.2 \%$ of participants reported being told by a mental health provider or physician that they had an eating disorder, including anorexia nervosa (3.2\%), bulimia nervosa (1.1\%), or other/not specified (1.1\%).

Mean scores, standard deviations, and percentile ranks for the EDE-Q subscales and Global score are presented in Table 2. Among cisgender bisexual plus men, 7.5\% scored in the clinically significant range on the Restraint, $2.2 \%$ on the Eating Concern, $12.9 \%$ on the Weight Concern, $19.4 \%$ on the Shape Concern subscales, and $2.2 \%$ on the Global score scale. In addition, $22.6 \%$ of cisgender bisexual plus men scored above the 2.48 cutoff score suggested by Machado et al. (2020) [22].

BMI was moderately positively correlated with the Eating Concern $(r=0.39, p<0.001)$ and Shape Concern $(r=0.40$, $p<0.001)$ subscales, as well as the Global score $(r=0.44$, $p<0.001)$. Also, BMI was strongly positively correlated with the Weight Concern $(r=0.52, p<0.001)$. No significant
Table 3 Proportion of cisgender bisexual plus women and men engaging in disordered eating behaviors in The PRIDE Study

\begin{tabular}{llllll}
\hline & \multicolumn{2}{l}{$\begin{array}{l}\text { Cisgender bisexual plus women } \\
(N=462)\end{array}$} & & \multicolumn{2}{l}{$\begin{array}{l}\text { Cisgender bisexual plus men } \\
(N=93)\end{array}$} \\
\cline { 2 - 3 } Disordered eating behavior & $\begin{array}{l}\text { Any occurrence } \\
(\%)\end{array}$ & $\begin{array}{l}\text { Regular occur- } \\
\text { rence }(\%)\end{array}$ & & $\begin{array}{l}\text { Any occurrence } \\
(\%)\end{array}$ & $\begin{array}{l}\text { Regular } \\
\text { occurrence } \\
(\%)\end{array}$ \\
\hline Dietary restraint & 19.3 & 5.6 & & 23.7 & 8.6 \\
Objective binge episodes & 11.1 & 3.7 & & 10.8 & 6.5 \\
Self-induced vomiting & 1.7 & 0.9 & & 0.0 & 0.0 \\
Laxative misuse & 0.4 & 0.4 & & 1.1 & 0.0 \\
Excessive exercise & 4.5 & 0.4 & & 5.4 & 2.2 \\
\hline
\end{tabular}

Regular occurrence of dietary restraint was defined as going for long periods of time $(8 \mathrm{~h})$ without eating anything to influence shape or weight for $\geq 13$ days over the past 28 days. Regular occurrence of excessive exercise was defined as exercising in a driven or compulsive way as a means of controlling weight, shape or amount of fat, or burning off calories for $\geq 20$ days over the past 28 days. For all other behaviors, regular occurrence was defined as $\geq 4$ occurrences over the past 28 days 
Table 4 Comparisons of eating attitudes and disordered eating behaviors in cisgender bisexual plus women $(N=462)$ to cisgender lesbian women $(N=476)$, and cisgender bisexual plus men $(N=93)$ to cisgender gay men $(N=978)$ from The PRIDE Study

\begin{tabular}{|c|c|c|c|c|c|c|c|c|c|}
\hline \multirow[b]{2}{*}{ Eating attitudes } & $\begin{array}{l}\text { Cisgender bisexual } \\
\text { plus women from } \\
\text { The PRIDE Study }\end{array}$ & $\begin{array}{l}\text { Cisgender lesbian } \\
\text { women from The } \\
\text { PRIDE Study }\end{array}$ & $t$-test & $p$ & $\begin{array}{l}\text { Cisgender bisexual } \\
\text { plus men from The } \\
\text { PRIDE Study }\end{array}$ & $\begin{array}{l}\text { Cisgender gay m } \\
\text { from The PRIDE } \\
\text { Study }\end{array}$ & & $t$-test & $p$ \\
\hline & \multicolumn{4}{|c|}{ Mean (standard deviation) } & \multicolumn{5}{|c|}{ Mean (standard deviation) } \\
\hline EDE-Q Restraint & $1.34(1.44)$ & $1.40(1.41)$ & -0.67 & 0.506 & $1.42(1.53)$ & $1.54(1.43)$ & & -0.73 & 0.469 \\
\hline EDE-Q EC & $0.96(1.13)$ & $0.80(1.05)$ & 2.20 & 0.028 & $0.63(0.96)$ & $0.63(0.98)$ & & 0.02 & 0.987 \\
\hline EDE-Q WC & $2.27(1.55)$ & $2.06(1.48)$ & 2.09 & 0.037 & $1.89(1.46)$ & $1.91(1.47)$ & & -0.12 & 0.902 \\
\hline EDE-Q SC & $2.42(1.62)$ & $2.22(1.57)$ & 1.94 & 0.053 & $2.30(1.57)$ & $2.41(1.62)$ & & -0.60 & 0.550 \\
\hline \multirow[t]{2}{*}{ EDE-Q Global } & $1.75(1.26)$ & $1.62(1.19)$ & 1.58 & 0.115 & $1.56(1.18)$ & $1.62(1.17)$ & & -0.47 & 0.642 \\
\hline & $\begin{array}{l}\text { Cisgender bisexual } \\
\text { plus women from } \\
\text { The PRIDE Study }\end{array}$ & $\begin{array}{l}\text { Cisgender lesbian } \\
\text { women from The } \\
\text { PRIDE Study }\end{array}$ & $Z$-test & $p$ & $\begin{array}{l}\text { Cisgender bisexual } \\
\text { plus men from The } \\
\text { PRIDE Study }\end{array}$ & $\begin{array}{l}\text { Cisgender gay } \\
\text { men from The } \\
\text { PRIDE Study }\end{array}$ & $Z$-test & $p$ & \\
\hline $\begin{array}{l}\text { Disordered eating } \\
\text { behaviors }\end{array}$ & Any occurrence (\%) & & & & Any occurrence (\%) & & & & \\
\hline Dietary restraint & 19.3 & 14.7 & 1.86 & 0.062 & 23.7 & 19.8 & 0.88 & & 380 \\
\hline $\begin{array}{l}\text { Objective binge } \\
\text { episodes }\end{array}$ & 11.1 & 10.1 & 0.48 & 0.634 & 10.8 & 10.9 & 0.06 & & 956 \\
\hline $\begin{array}{l}\text { Self-induced vomit- } \\
\text { ing }\end{array}$ & 1.7 & 0.4 & - & $0.062^{\dagger}$ & - & 0.6 & - & - & \\
\hline Laxative misuse & 0.4 & 0.2 & - & $0.620^{\dagger}$ & 1.1 & 1.1 & - & & $.999^{\dagger}$ \\
\hline Excessive exercise & 4.5 & 5.9 & 0.92 & 0.358 & 5.4 & 10.1 & 1.48 & & 140 \\
\hline
\end{tabular}

$E D E-Q$ Eating Disorder Examination-Questionnaire, $E D E-Q E C$ Eating Concern subscale, $E D E-Q W C$ Weight Concern subscale, $E D E-Q S C$ Shape Concern subscale, EDE- $Q$ Global Global score

Any occurrence was defined as $\geq 1$ episode in the past 28 days. EDE-Q scores were compared using independent samples $t$-tests. Proportions of disordered eating behaviors were compared with Z-tests or Fisher's exact tests

†Fisher's exact test

$* p<.005$ (after Bonferroni correction)

correlation was observed between participants' BMI and the Restraint subscale $(r=0.19, p=0.073)$.

Any occurrence and regular occurrences of disordered eating behaviors are presented in Table 3 . Nearly one quarter of participants reported any dietary restraint during the previous 28 days. Close to $10 \%$ of the participants endorsed at least one episode of objective binge eating and around 5\% reported at least one episode of excessive exercise over the past 28 days. Episodes of self-induced vomiting were not observed, and any laxative misuse in the last 28 days was reported in $1.1 \%$ of the cases $(n=1)$.

Table 4 shows comparisons in the EDE-Q subscales, the EDE-Q Global score, and disordered eating behaviors between cisgender bisexual plus men and cisgender gay men. No differences were observed in either attitudinal subscales or key ED behaviors across groups.

Table 5 shows group comparison in EDE-Q subscales, Global score, and disordered eating behaviors between age-matched cisgender bisexual plus men from The PRIDE Study with presumed cisgender heterosexual men from previously published norms [15]. Cisgender bisexual plus men had a higher Weight Concern subscale score (2.26) than presumed cisgender heterosexual men previously published (1.29). Attitudinal scores in cisgender bisexual plus men compared to presumed cisgender heterosexual men and cisgender men included Eating Concern ( 0.88 vs 0.43 , $p=0.021)$, Shape Concern (2.44 vs $1.59, p=0.014)$, and Global score (1.74 vs $1.09, p=0.009)$; however, these did not reach the $p<0.005$ threshold after the Bonferroni correction. In terms of disordered eating behaviors, no significant differences were observed for dietary restraint, objective binge episodes, and excessive exercise. Contrary to presumed cisgender heterosexual men, cisgender bisexual plus men did not report any occurrence of self-induced vomiting and laxative misuse in the past 28 days.

Table 6 shows group comparisons in EDE-Q subscales, the EDE-Q Global score, and key ED behaviors between cisgender bisexual plus women and men. With the exception of the Eating Concern subscale, where cisgender bisexual plus women showed higher levels than cisgender bisexual plus men, no differences were observed across attitudinal and behavioral items of the EDE-Q between cisgender bisexual plus women and men. 


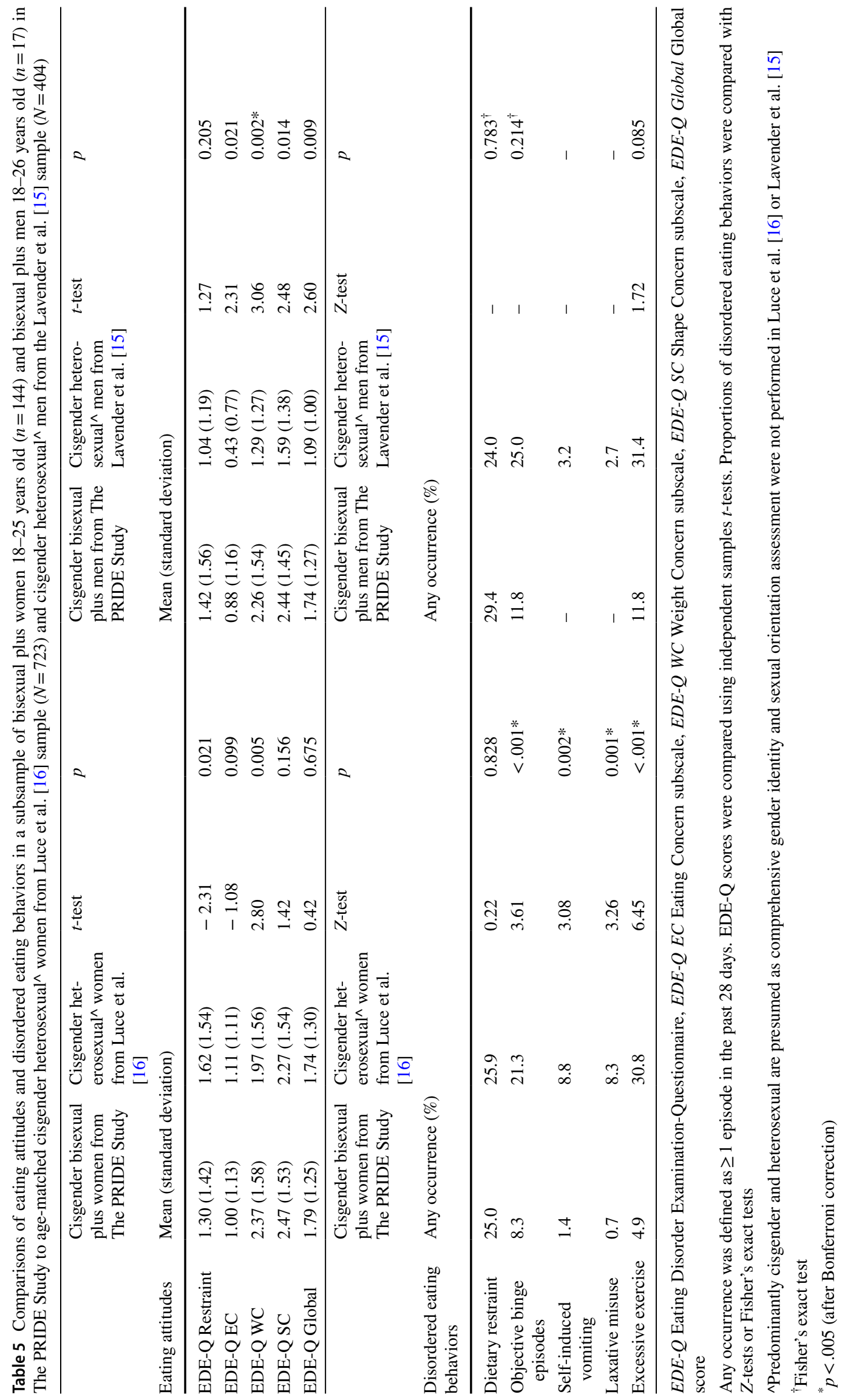


Table 6 Comparisons of eating attitudes and disordered eating behaviors in cisgender bisexual plus women $(N=462)$ and cisgender bisexual plus men $(N=93)$ from The PRIDE Study

\begin{tabular}{|c|c|c|c|c|}
\hline Eating attitudes & $\begin{array}{l}\text { Cisgender bisexual plus women } \\
\text { M (SD) }\end{array}$ & $\begin{array}{l}\text { Cisgender bisexual plus men } \\
\mathrm{M}(\mathrm{SD})\end{array}$ & $t$-test & $p$ \\
\hline EDE-Q Restraint & $1.34(1.44)$ & $1.42(1.53)$ & -0.51 & 0.611 \\
\hline EDE-Q EC & $0.96(1.13)$ & $0.63(0.96)$ & 2.64 & 0.008 \\
\hline EDE-Q WC & $2.27(1.55)$ & $1.89(1.46)$ & 2.18 & 0.030 \\
\hline EDE-Q SC & $2.42(1.62)$ & $2.30(1.57)$ & 0.67 & 0.504 \\
\hline EDE-Q Global & $1.75(1.26)$ & $1.56(1.18)$ & 1.33 & 0.185 \\
\hline Disordered eating behaviors & $\begin{array}{l}\text { Cisgender bisexual plus women } \\
\text { Any occurrence }(\%)\end{array}$ & $\begin{array}{l}\text { Cisgender bisexual plus men } \\
\text { Any occurrence }(\%)\end{array}$ & $Z$-test & $p$ \\
\hline Dietary restraint & 19.3 & 23.7 & 0.97 & 0.334 \\
\hline Objective binge episodes & 11.1 & 10.8 & 0.08 & 0.936 \\
\hline Self-induced vomiting & 1.7 & - & - & - \\
\hline Laxative misuse & 0.4 & 1.1 & - & $0.426^{\dagger}$ \\
\hline Excessive exercise & 4.5 & 5.4 & - & $0.788^{\dagger}$ \\
\hline
\end{tabular}

$M$ mean, $S D$ standard deviation, $E D E-Q$ Eating Disorder Examination-Questionnaire, $E D E-Q E C$ Eating Concern subscale, $E D E-Q W C$ Weight Concern subscale, EDE- $Q S C$ Shape Concern subscale, EDE- $Q$ Global Global score

Any occurrence was defined as $\geq 1$ episode in the past 28 days. EDE-Q scores were compared using independent samples $t$-tests. Proportions of disordered eating behaviors were compared with Z-tests or Fisher's exact tests

†Fisher's exact test

${ }^{*} p<.005$ (after Bonferroni correction)

\section{Discussion}

We describe community norms for the EDE-Q for the first time among cisgender bisexual plus women and men. Establishing norms for cisgender bisexual plus women and men is important because bisexual plus people are often not delineated from other sexual minority populations (e.g., gay or lesbian), but may have unique health considerations, including eating disorders $[1,31]$. Furthermore, most prior community norms of the EDE-Q in women and men did not assess sexual orientation and were presumably predominantly cisgender heterosexual samples [15, 16, 28, 32-36]. A lower percentage of cisgender bisexual plus women reported any occurrence of objective binge episodes, self-induced vomiting, laxative misuse, and excessive exercise than presumed cisgender heterosexual women previously published [16]. Cisgender bisexual plus men had a higher Weight Concern subscale score than presumed cisgender heterosexual men previously published [15].

\section{Cisgender bisexual plus women}

Cisgender bisexual plus women reported the highest scores on the Shape Concern and Weight Concern subscales, consistent with previous EDE-Q norms in presumably cisgender heterosexual young women $[16,28]$. The most common disordered eating behaviors reported by cisgender bisexual plus women were dietary restraint (19\%) and objective binge episodes (11\%). Interestingly, we found lower rates of objective binge episodes, self-induced vomiting, laxative misuse, and excessive exercise in cisgender bisexual plus women compared to presumed cisgender heterosexual women previously published [16]. In a prior study, fasting $>24 \mathrm{~h}$ were reported among heterosexual (13\%), bisexual (26\%), and lesbian (28\%) girls [37]. Another study reported binge eating rates in heterosexual (4\%), bisexual (10\%), and lesbian (7\%) women 19-20 years old [38], similar to the rates of binge eating found in this study. However, both studies used heterosexual girls or women as the reference group and thus did not statistically test for differences between bisexual and lesbian girls or women [37, 38]. We did not find significant differences between cisgender bisexual plus women and cisgender lesbian women in EDE-Q Global or subscale scores or disordered eating behaviors.

\section{Cisgender bisexual plus men}

Among cisgender bisexual plus men, we found that the Shape Concern and Weight Concern scores were the highest among all the subscale scores, similar to cisgender bisexual plus women in the present study. These were also the highest subscales previously reported among cisgender gay men [19] and presumed cisgender heterosexual men [15]. Cisgender bisexual plus men in this sample reported higher Weight Concern subscale scores than presumed cisgender 
heterosexual men previously published [15]. Nearly a quarter of our sample's cisgender bisexual plus men reported dietary restraint and one tenth reported objective binge episodes in the past 28 days. Laxative misuse and self-induced vomiting were infrequent occurrences. BMI was significantly correlated with the EDE-Q subscales in bisexual plus men, similar to findings previously shown among cisgender gay men [19] and presumed cisgender heterosexual men [15]. We did not find significant differences between cisgender bisexual plus men and cisgender gay men in EDE-Q Global or subscale scores or disordered eating behaviors. These results are consistent with the growing literature demonstrating eating disorder concerns in sexual minority boys and men $[31,37,39,40]$, and add to the literature by providing estimates of eating disorder attitudes and disordered eating behaviors specific to cisgender bisexual plus men.

\section{Gender differences}

There were not significant gender differences in the prevalence of disordered eating behaviors among cisgender bisexual plus women and men. Prior studies among presumed cisgender and heterosexual women [16] and men [15] undergraduate students found that women had higher rates of purging (laxatives and self-induced vomiting) than men [41, 42]. In these same studies, women had higher scores on the EDE-Q Global score and all subscale scores compared to men. In contrast, we did not find significant differences in the EDE-Q Global score or subscale scores among cisgender bisexual plus women and men, except for a higher Eating Concern score among cisgender bisexual plus women compared to men. The lack of gender differences in disordered eating behaviors among cisgender bisexual plus populations highlights some of the unique eating disorder considerations in these populations.

\section{Minority stress theory}

Eating disorder attitudes and disordered eating behaviors among cisgender bisexual plus women and men may in part be explained by the minority stress theory [43], which includes specific biphobic stressors [1]. The minority stress theory posits that prejudice, stigma, and discrimination experienced by sexual minorities, including bisexual plus people, may lead to health disparities. Furthermore, biphobia represents the additional prejudice, stigma, and discrimination experienced by bisexual plus individuals from people with monosexual orientations, including those who identify as gay, lesbian, or heterosexual [1]. Bisexual plus people may face microaggressions, macroaggressions, or bullying from people in the gay and lesbian community due to the assumption that bisexual people may escape some discrimination by heterosexual people by conforming to social expectations of opposite-gender sexual activity and romance [44]. Bisexual plus people may cope with minority stress and biphobia through disordered eating behaviors. The present study results may be consistent with minority stress particularly for cisgender bisexual plus men who reported higher Weight Concern subscales than presumed cisgender heterosexual men.

\section{Limitations and strengths}

There are some limitations to consider in this study. The EDE-Q is based on self-report which may lead to reporting bias. For the purposes of this study, we included cisgender people who identified as bisexual, pansexual, and polysexual, which may obscure possible differences across these groups. We did not include cisgender individuals who identified as "queer." Some people who identify as queer may see themselves as having a type of bisexual plus identity; others may not. Therefore, we made the a priori decision to exclude these participants; however, identifying eating disorder attitudes and behaviors in people who identify as queer could be an area of future research. Our sample was self-selected, recruited online, highly educated, and mostly white, which limits generalizability as people of color make up a significant portion of the bisexual plus community [45]. Although we were able to assess group comparisons between cisgender lesbian and bisexual plus women, as well as between cisgender gay and bisexual plus men, The PRIDE Study focuses recruitment on sexual and gender minority populations and, thus, we did not recruit cisgender heterosexual women and men for group comparisons. To address this, we identified previously published samples of presumed cisgender heterosexual women [16] and men [15] for comparison; however, these were in young adult university students. Thus, we developed EDE-Q norms for age-matched subsamples of cisgender bisexual plus men and women. Although our age-matched sample of cisgender bisexual plus men was small $(N=17)$, we nonetheless were able to detect a statistically significant difference in the Weight Concern subscale, even after the Bonferroni correction $(p<0.005)$. We did not collect clinical interview data to establish an eating disorder diagnosis and thus were unable to establish clinical cut-offs specific to cisgender bisexual plus women and men, but we did use previously published empirically-derived cutoffs [22]. Despite these limitations, leveraging The PRIDE Study allowed us to describe eating disorder phenomenology in a large number of cisgender bisexual plus people, an understudied sexual minority group. 


\section{Conclusion}

We report for the first time EDE-Q norms among cisgender bisexual plus women and men. These findings can help researchers and clinicians to interpret EDE-Q scores in this understudied sexual minority population. Clinicians should be aware that cisgender bisexual plus people may engage in disordered eating behaviors and should consider screening for these behaviors. Future research could examine how factors such as race/ethnicity, age, and socioeconomic status may influence eating disorders and the EDE-Q among cisgender bisexual plus people. In addition, the EDE-Q does not assess for muscularity-oriented concerns and this is an area of future research related to body image in sexual minority populations.

\section{What is already known on this subject?}

Although cisgender bisexual plus women and men may be at elevated risk for eating disorders, community norms of the Eating Disorders Examination Questionnaire (EDE-Q) in these populations are currently lacking.

\section{What does this study add?}

In a large sample of bisexual plus women and men in the US, mean and standard deviation EDE-Q Global score were $1.75 \pm 1.26$ and $1.56 \pm 1.18$, while $27.5 \%$ and $22.6 \%$ scored in the clinically significant range, respectively.

\begin{abstract}
Acknowledgements The authors thank Samuel Benabou for editorial assistance. The PRIDE Study is a community-engaged research project that serves and is made possible by LGBTQ+ community involvement at multiple points in the research process, including the dissemination of findings. We acknowledge the courage and dedication of The PRIDE Study participants for sharing their stories, the careful attention of PRIDEnet Participant Advisory Committee (PAC) members for reviewing and improving every study application, and the enthusiastic engagement of PRIDEnet Ambassadors and Community Partners for bringing thoughtful perspectives as well as promoting enrollment and disseminating findings. For more information, please visit https://pride study.org/pridenet.
\end{abstract}

Funding $\mathrm{JN}$ is supported by the American Heart Association Career Development Award (CDA34760281) and was a participant in the Pediatric Scientist Development Program (K12 HD000850) supported by the American Academy of Pediatrics and the American Pediatric Society. MC was supported by a Clinical Research Training Fellowship from the American Academy of Neurology and the Tourette Association of America. SBM was supported by the National Institutes of Health (K23 MH115184). JOM was partially supported by K12DK111028 from the National Institute of Diabetes, Digestive, and Kidney Disorders. AF was supported by the National Institute on Drug Abuse (K23DA039800). Research reported in this article was partially funded through a Patient-Centered Outcomes Research Institute (www. pcori.org; PPRN-1501-26848) to MRL. The statements in this article are solely the responsibility of the authors and do not necessarily represent the views of Patient-Centered Outcomes Research Institute, its Board of Governors or Methodology Committee, or the National Institutes of Health.

\section{Compliance with ethical standards}

Conflict of interest Dr. Juno Obedin-Maliver has consulted for Sage Therapeutics (5/2017) in a one-day advisory board, Ibis Reproductive Health (a non-for-profit research group 3/2017-5/2018), Hims, Inc. (2019-present) and Folx, Inc (2020 - present). Dr. Mitchell R. Lunn has consulted for Hims, Inc. (2019-present) and Folx, Inc (2020present). None of these roles present a conflict of interest with this work as described here. The other authors have no conflicts of interest to report.

Ethical approval The University of California, San Francisco Institutional Review Board approved this study on 2 February 2018 (\#1621213). All procedures performed in this study were in accordance with the ethical standards of the university's Institutional Review Board and with the 1964 Helsinki Declaration and its later amendments or comparable ethical standards.

Informed consent Written informed consent was obtained from all participants.

Open Access This article is licensed under a Creative Commons Attribution 4.0 International License, which permits use, sharing, adaptation, distribution and reproduction in any medium or format, as long as you give appropriate credit to the original author(s) and the source, provide a link to the Creative Commons licence, and indicate if changes were made. The images or other third party material in this article are included in the article's Creative Commons licence, unless indicated otherwise in a credit line to the material. If material is not included in the article's Creative Commons licence and your intended use is not permitted by statutory regulation or exceeds the permitted use, you will need to obtain permission directly from the copyright holder. To view a copy of this licence, visit http://creativecommons.org/licenses/by/4.0/.

\section{References}

1. Friedman MR, Dodge B, Schick V et al (2014) From bias to bisexual health disparities: attitudes toward bisexual men and women in the united states. LGBT Heal 1:309-318. https://doi.org/10.1089/ lgbt.2014.0005

2. Feinstein BA, Xavier Hall CD, Dyar C, Davila J (2020) Motivations for sexual identity concealment and their associations with mental health among bisexual, pansexual, queer, and fluid (Bi+) individuals. J Bisex. https://doi.org/10.1080/15299 716.2020 .1743402

3. McClelland SI, Rubin JD, Bauermeister JA (2016) Adapting to Injustice. Psychol Women Q 40:532-550. https://doi. org/10.1177/0361684316664514

4. Bostwick W, Hequembourg A (2014) "Just a little hint": bisexualspecific microaggressions and their connection to epistemic injustices. Cult Heal Sex 16:488-503. https://doi.org/10.1080/13691 058.2014.889754

5. Sarno E, Wright AJ (2013) Homonegative microaggressions and identity in bisexual men and women. J Bisex 13:63-81. https:// doi.org/10.1080/15299716.2013.756677

6. Meneguzzo P, Collantoni E, Gallicchio D et al (2018) Eating disorders symptoms in sexual minority women: a systematic review. 
Eur Eat Disord Rev J Eat Disord Assoc 26:275-292. https://doi. org/10.1002/erv.2601

7. Nagata JM, Ganson KT, Austin SB (2020) Emerging trends in eating disorders among sexual and gender minorities. Curr Opin Psychiatry 33(6):562-567

8. Henn AT, Taube CO, Vocks S, Hartmann AS (2019) Body image as well as eating disorder and body dysmorphic disorder symptoms in heterosexual, homosexual, and bisexual women. Front Psychiatry 10:531. https://doi.org/10.3389/fpsyt.2019.00531

9. Bayer V, Robert-McComb JJ, Clopton JR, Reich DA (2017) Investigating the influence of shame, depression, and distress tolerance on the relationship between internalized homophobia and binge eating in lesbian and bisexual women. Eat Behav 24:39-44. https ://doi.org/10.1016/j.eatbeh.2016.12.001

10. Gigi I, Bachner-Melman R, Lev-Ari L (2016) The association between sexual orientation, susceptibility to social messages and disordered eating in men. Appetite 99:25-33. https://doi. org/10.1016/j.appet.2015.12.027

11. Lunn MR, Cui W, Zack MM et al (2017) Sociodemographic characteristics and health outcomes among lesbian, gay, and bisexual US adults using healthy people 2020 leading health indicators. LGBT Heal 4:283-294. https://doi.org/10.1089/lgbt.2016.0087

12. Bankoff SM, Richards LK, Bartlett B et al (2016) Examining weight and eating behavior by sexual orientation in a sample of male veterans. Compr Psychiatry 68:134-139. https://doi. org/10.1016/j.comppsych.2016.03.007

13. Von Schell A, Ohrt TK, Bruening AB, Perez M (2018) Rates of disordered eating behaviors across sexual minority undergraduate men and women. Psychol Sex Orientat Gend Divers 5:352-359. https://doi.org/10.1037/sgd0000278

14. Davids CM, Green MA (2011) A preliminary investigation of body dissatisfaction and eating disorder symptomatology with bisexual individuals. Sex Roles 65:533. https://doi.org/10.1007/ s11199-011-9963-y

15. Lavender JM, De YKP, Anderson DA (2010) Eating disorder examination questionnaire (EDE-Q): norms for undergraduate men. Eat Behav 11:119-121. https://doi.org/10.1016/j.eatbe h.2009.09.005

16. Luce KH, Crowther JH, Pole M (2008) Eating disorder examination questionnaire (EDE-Q): norms for undergraduate women. Int J Eat Disord 41:273-276. https://doi.org/10.1002/eat.20504

17. Lunn MR, Lubensky M, Hunt C et al (2019) A digital health research platform for community engagement, recruitment, and retention of sexual and gender minority adults in a national longitudinal cohort study-The PRIDE Study. J Am Med Inf Assoc JAMIA. https://doi.org/10.1093/jamia/ocz082

18. Lunn MR, Capriotti MR, Flentje A et al (2019) Using mobile technology to engage sexual and gender minorities in clinical research. PLoS ONE 14:e0216282. https://doi.org/10.1371/journ al.pone. 0216282

19. Nagata JM, Capriotti MR, Murray SB et al (2020) Community norms for the eating disorder examination questionnaire among cisgender gay men. Eur Eat Disord Rev J Eat Disord Assoc 28:92101. https://doi.org/10.1002/erv.2708

20. Nagata JM, Murray SB, Flentje A et al (2020) Eating disorder attitudes and disordered eating behaviors as measured by the Eating Disorder Examination Questionnaire (EDE-Q) among cisgender lesbian women. Body Image 34:215-220. https://doi. org/10.1016/j.bodyim.2020.06.005

21. Fairburn CG, Beglin S (2008) Eating disorder examination questionnaire. In: Fairburn CG (ed.). Guilford Press, New York. pp 309-313

22. Machado PPP, Grilo CM, Rodrigues TF et al (2020) Eating disorder examination - questionnaire short forms: a comparison. Int J Eat Disord 53:937-944. https://doi.org/10.1002/eat.23275
23. Nagata JM, Murray SB, Compte EJ et al (2020) Community norms for the Eating Disorder Examination Questionnaire (EDEQ) among transgender men and women. Eat Behav. https://doi. org/10.1016/j.eatbeh.2020.101381

24. Kelly NR, Cotter EW, Mazzeo SE (2012) Eating Disorder Examination Questionnaire (EDE-Q): norms for black women. Eat Behav 13:429-432. https://doi.org/10.1016/j.eatbeh.2012.09.001

25. Villarroel AM, Penelo E, Portell M, Raich RM (2011) Screening for eating disorders in undergraduate women: norms and validity of the spanish version of the eating disorder examination questionnaire (EDE-Q). J Psychopathol Behav Assess 33:121-128. https ://doi.org/10.1007/s10862-009-9177-6

26. Machado PPP, Martins C, Vaz AR et al (2014) Eating disorder examination questionnaire: psychometric properties and norms for the Portuguese population. Eur Eat Disord Rev 22:448-453. https://doi.org/10.1002/erv.2318

27. Nagata JM, Compte EJ, Cattle CJ et al (2020) Community norms for the Eating Disorder Examination Questionnaire (EDE-Q) among gender-expansive populations. J Eat Disord 27:101381. https://doi.org/10.1186/s40337-020-00352-x

28. Mond JM, Hay PJ, Rodgers B, Owen C (2006) Eating Disorder Examination Questionnaire (EDE-Q): norms for young adult women. Behav Res Ther 44:53-62. https://doi.org/10.1016/j. brat.2004.12.003

29. American Psychiatric Association (2013) Diagnostic and statistical manual of mental disorders, 5th edn. American Psychiatric Publishing, Arlington

30. Cohen J (1988) Statistical power analysis for the behavioral sciences, 2nd edn. Lawrence Erlbaum Associates, Hillsdale

31. Tabler JT, Schmitz R, Geist C, Nagata JM (2019) Does it get better? Change in depressive symptoms from late-adolescence to early-adulthood, disordered eating behaviors, and sexual identity. J Gay Lesbian Ment Health 23:221-243. https://doi. org/10.1080/19359705.2019.1568944

32. Hilbert A, de Zwaan M, Braehler E (2012) How frequent are eating disturbances in the population? Norms of the eating disorder examination-questionnaire. PLoS ONE 7:e29125. https://doi. org/10.1371/journal.pone.0029125

33. Quick VM, Byrd-Bredbenner C (2013) Eating Disorders Examination Questionnaire (EDE-Q): norms for US college students. Eat Weight Disord EWD 18:29-35. https://doi.org/10.1007/s4051 9-013-0015-1

34. Reas DL, Øverås M, Rø O (2012) Norms for the Eating Disorder Examination Questionnaire (EDE-Q) among high school and university men. Eat Disord 20:437-443. https://doi. org/10.1080/10640266.2012.715523

35. Rø O, Reas DL, Lask B (2010) Norms for the eating disorder examination questionnaire among female university students in Norway. Nord J Psychiatry 64:428-432. https://doi. org/10.3109/08039481003797235

36. Darcy AM, Hardy KK, Lock J et al (2013) The eating disorder examination questionnaire (EDE-Q) among university men and women at different levels of athleticism. Eat Behav 14:378-381. https://doi.org/10.1016/j.eatbeh.2013.04.002

37. Hadland SE, Austin SB, Goodenow CS, Calzo JP (2014) Weight misperception and unhealthy weight control behaviors among sexual minorities in the general adolescent population. J Adolesc Health 54:296-303. https://doi.org/10.1016/j.jadohealth .2013.08.021

38. Austin SB, Ziyadeh NJ, Corliss HL et al (2009) Sexual orientation disparities in purging and binge eating from early to late adolescence. J Adolesc Heal Off Publ Soc Adolesc Med 45:238-245. https://doi.org/10.1016/j.jadohealth.2009.02.001

39. Calzo JP, Austin SB, Micali N (2018) Sexual orientation disparities in eating disorder symptoms among adolescent boys and girls 
in the UK. Eur Child Adolesc Psychiatry 27:1483-1490. https:// doi.org/10.1007/s00787-018-1145-9

40. Watson RJ, Adjei J, Saewyc E et al (2017) Trends and disparities in disordered eating among heterosexual and sexual minority adolescents. Int J Eat Disord 50:22-31. https://doi.org/10.1002/ eat. 22576

41. Nagata JM, Garber AK, Tabler JL, Murray SB, Bibbins-Domingo K (2018) Prevalence and correlates of disordered eating behaviors among young adults with overweight or obesity. J Gen Int Med 33(8):1337-1343

42. Nagata JM, Domingue BW, Darmstadt GL, Weber AM, Meausoone V, Cislaghi B, Shakya HB (2020) Gender norms and weight control behaviors in U.S. adolescents: a prospective cohort study (1994-2002). J Adolesc Health 66(1):S34-S41
43. Meyer IH (2003) Prejudice, social stress, and mental health in lesbian, gay, and bisexual populations: conceptual issues and research evidence. Psychol Bull 129:674-697. https://doi. org/10.1037/0033-2909.129.5.674

44. Roffee JA, Waling A (2016) Rethinking microaggressions and anti-social behaviour against LGBTIQ+ youth. Safer Commun 15:190-201. https://doi.org/10.1108/SC-02-2016-0004

45. Gates GJ (2010) Sexual minorities in the 2008 general social survey: coming out and demographic characteristics. Los Angeles, $\mathrm{CA}$

Publisher's Note Springer Nature remains neutral with regard to jurisdictional claims in published maps and institutional affiliations. 